\title{
Malaria surge feared
}

\section{The WHO releases action plan to tackle the spread of insecticide-resistant mosquitoes.}

\section{BY AMY MAXMEN}

$\mathrm{T}$ he war to bring malaria to heel has made slow but steady progress during the past decade, with the overall mortality rate dropping by more than $25 \%$ since 2000 . A key factor in this progress has been improved control of mosquitoes, which transmit the Plasmodium parasite - a potent killer that claimed an estimated 655,000 lives in 2010 alone. But health officials fear that the spread of insecticideresistant mosquitoes could bring about a resurgence of the disease. To help combat this threat, on 15 May the World Health Organization (WHO), based in Geneva, Switzerland, issued a strategic plan to curb the spread of resistance.

"We don't want to wait for failures to happen," says David Brandling-Bennett, the senior adviser for infectious diseases at the Bill \& Melinda Gates Foundation in Seattle, Washington, who advised on the document.

Such failures could reverse the recent drop in malaria mortality credited to insecticide spraying in the home and coating of bed nets, which save about 220,000 children's lives each year, according to the WHO. Insecticide resistance could also result in as many as 26 million further cases a year, the organization predicts, costing an extra US\$30 million to \$60 million annually for tests and medicines.

The WHO report says that insecticideresistant mosquitoes already inhabit 64 malariaridden countries (see map). The problem is particularly acute in sub-Saharan African countries such as Benin, Burkina Faso, Cameroon, Côte d'Ivoire, Ghana, Ethiopia and Uganda, where mosquitoes are frequently resistant to compounds known as pyrethroids and even

"In 2004, there
were pockets
of resistance
in Africa, and
now there are
pockets of
susceptibility."

to the organochloride DDT, venerable tools of mosquito control. Because they are extremely safe for children, effective against mosquitoes and affordable, pyrethroids are the only insecticides used to treat bed nets, as well as the first choice for household spraying.

Health authorities in Somalia, Sudan and Turkey have also reported sporadic resistance to the two other classes of insecticides recommended by the WHO for safe and effective household spraying: carbamates and organophosphates. Resistance has probably evolved several times independently, and is now spreading as extensive use of pyrethroids and

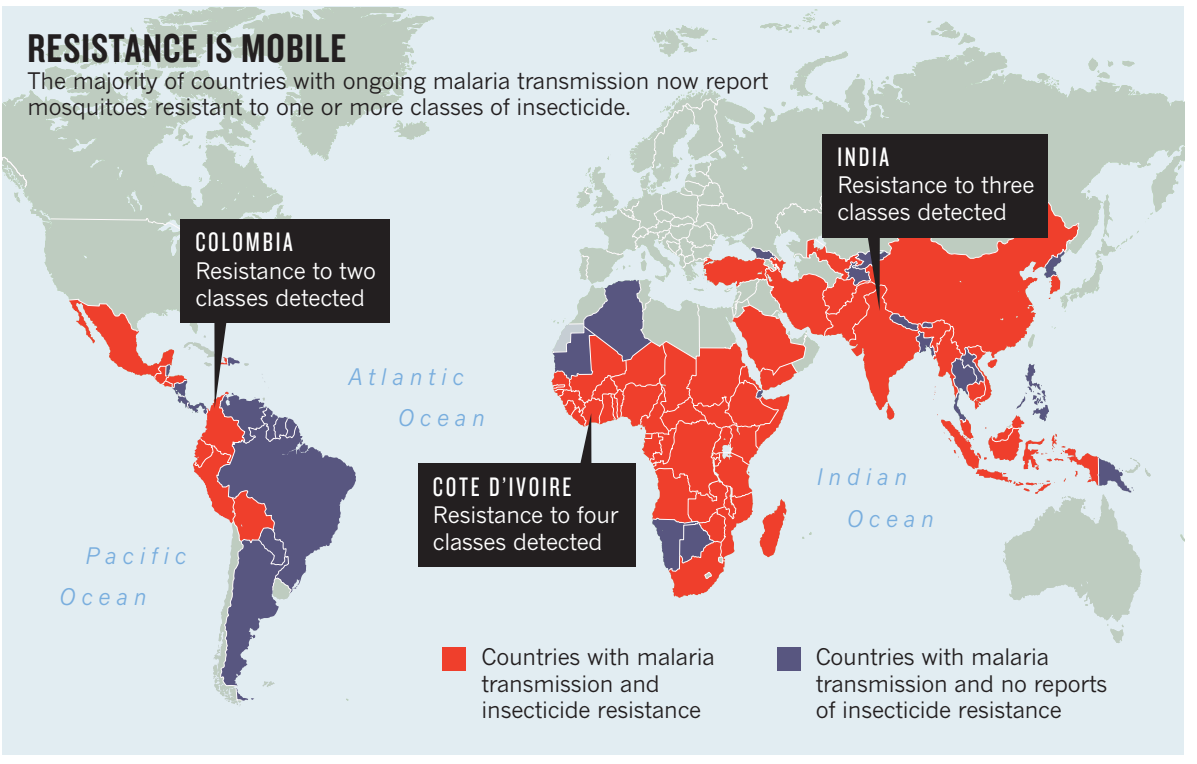

other insecticides favours resistant mosquitoes. "In 2004, there were pockets of resistance in Africa, and now there are pockets of susceptibility," says Janet Hemingway, chief executive of the Innovative Vector Control Consortium (IVCC), a product-development partnership based in the United Kingdom.

Among other things, the WHO recommends rotating the classes of pesticides used to spray houses, and developing safe and effective non-pyrethroid insecticides that can be used to treat bed nets. To implement all of the WHO's suggestions would cost $\$ 200$ million - on top of the $\$ 6$ billion that the WHO requested last year to fund existing malaria-control programmes. Rob Newman, director of the Global Malaria Programme at the WHO, hopes that the report will draw more funds to the table as donors grasp the situation. "If we can stop pyrethroid resistance from spreading, it will be cheaper in the long run," Newman says.

But the two largest players in malaria aid the Global Fund to Fight AIDS, Tuberculosis and Malaria, and the US President's Malaria Initiative (PMI) - have not yet pledged additional money to fight resistance. Their spending on mosquito control is already high - in $2009,39 \%$ of the Global Fund's malaria expenditures went towards insecticide-treated bed nets and household spraying, as did $59 \%$ of the

\section{DNATURE.COM}

To read an Outlook on malaria, see: go.nature.com/gzyguu nets, and where spraying is concerned they are less costly than the alternatives. Vestergaard Frandsen, a company based in Lausanne, Switzerland, says that it has in the pipeline a bed net coated with a non-pyrethroid insecticide - one that does not belong to any of the four WHO-approved classes - and that the company expects to bring this to market within the next five years. It is also one of several companies partnering with the IVCC to create innovative mosquito-control products.

In the meantime, health officials may be able to keep malaria at bay by swapping insecticides. The report notes that in Colombia, for instance, mosquitoes regained susceptibility to pyrethroids after five years of treatment with an organophosphate. But some African countries lack the surveillance needed to spur such an approach. To address that deficiency, the report urges that a global database be set up to track the spread of resistance, and that entomologists be trained and hired at surveillance stations. That could prove the most challenging goal of all. "Nobody wants to fund capacity building," says Newman. "Donors would rather say they purchased $\$ 10,000$ in bed nets than pay a salary."

African ministers of health realize the need to manage resistance but can't do much without outside funds, explains Maureen Coetzee, a medical entomologist at the University of the Witwatersrand in Johannesburg, South Africa. "In some countries, malaria control means one person sitting in one room, and he's lucky if he's got a chair," she says. 